\title{
COMPORTAMIENTO CON DIFERENTES ENMIENDAS ORGÁNICAS DEL NEMATODO DEL NÓDULO Meloidogyne incognita (Kofoid and White, 1919), Chitwood1949, EN PIMIENTO PAPRIKA (Capsicum annum L.)
}

\author{
BEHAVIOR WITH DIFFERENT ORGANIC AMENDMENTS nodule nematode \\ Meloidogyne incognita (Kofoid and White, 1919) Chitwood 1949, BELL PEPPER \\ (Capsicum annuum L.)
}

${ }^{1}$ Paola Revilla Cervantes y ${ }^{2}$ Angel Alfonso Palomo Herrera

\begin{abstract}
Resumen
Se evaluó el efecto de la incorporación de 8 diferentes enmiendas orgánicas sobre sustrato inoculado y sin inoculación de Meloidogyne incognita, en pimiento Páprika (Capsicumannuum). El experimento se desarrolló en el invernadero de Nematología de la UNALM. Los tratamientos sin inoculación se establecieron con la finalidad de determinar si existe efecto fitotóxico de las enmiendas orgánicas. Se empleó un diseño completamente aleatorio con 4 repeticiones por tratamiento. Los resultados muestran que las enmiendas orgánicas rastrojo de nabo y holantao redujeron las poblaciones de $M$. incognita y la nodulación en las raíces. Los parámetros de crecimiento de la planta que se vieron favorecidos fueron: peso fresco de parte aérea y volumen de raíz, incorporando las enmiendas guano de vacuno, rastrojo de holantao y guano de ave respectivamente.
\end{abstract}

Palabras clave: enmiendas orgánicas, Meloidogyne incognita, PapriQueen.

\begin{abstract}
The effect of the addition of eight different organic amendments on inoculated substrate and without inoculation of Meloidogyne incognita in pepper Paprika (Capsicum annuum) was tested.The experiment was conducted in the greenhouse of Nematology at UNALM.Without inoculation treatments were established in order to determine if phytotoxic effect of organic amendments exists. We used a completely randomized design with four replicates per treatment. The results show that organic amendments: holantao stubble and turnip stubble reduced populations of M. incognita. and galls on the roots. Plant growth parameters that were favored with amendments incorporated are: aerial fresh weight and root volume, with the incorporation of cattle manure, holantao stubble and poultry manure, respectively.
\end{abstract}

Key words: organic amendments, Meloidogyne incognita, Papriqueen.

\section{Introducción}

El uso de nematicidas químicos viene siendo el método de control de nematodos más utilizado por los agricultores; por ello, la búsqueda de alternativas sostenibles y de menor costo para el control de nematodos se hace necesaria, planteándose el empleo de diversas enmiendas orgánicas con efecto nematicida o nematostático como una alternativa de solución.

La reducción en las densidades poblacionales de nematodos fitoparásitos en respuesta a la aplicación de enmiendas orgánicas ha sido reportada en muchos estudios (Muller y Gooch, 1982). Existen plantas que contienen ciertos químicos, tales como el amoníaco, nitritos, sulfuros de hidrógeno, ácidos orgánicos $\mathrm{y}$ otras sustancias químicas que se producen a partir de la materia orgánica (M.O.) y pueden ser directamente nematicidas (Hollis and Rodriguez-Kabana, 1966; Badra and Eligindi, 1979). También existen compuestos azufrados de origen vegetal que tienen una distribución más restringida, como los compuestos GLU en las crucíferas (J. Auger, E. Thibout, 2004).

\footnotetext{
${ }^{1}$ Ingeniero Agrónomo. E-mail: paorevillacer@gmail.com

${ }^{2}$ Departamento Fitopatología, Facultad Agronomía - UNALM, Lima, Perú. E-mail: fonz@lamolina.edu.pe
} 
Los abonos orgánicos aplicados al suelo también mejoran la condición de la raíz y aportan nutrientes a la planta, lo que favorece un crecimiento adecuado del cultivo que le permite tolerar las enfermedades o escapar de la infección (Huber, 1991). Además, la adición de M.O. al suelo estimula la actividad de bacterias, hongos, algas y otros agentes de biocontrol del suelo, proporcionando alimento para su establecimiento y actividad (Hoitink y Grebus, 1994; Craft y Nelson, 1996; Hoitink y Boehm, 2001; Noble y Coventry, 2005).

En la búsqueda de alternativas de control de Meloidogyne incognita, el presente estudio tiene como objetivo determinar el efecto de diferentes enmiendas orgánicas en el comportamiento de Meloidogyneincognita en pimiento Páprika (Capsicumannuum L.) a nivel de invernadero.

\section{Materiales y Métodos}

Instalación de Plantas en el Invernadero

El ensayo se realizó en el invernadero de Fitopatología de la Universidad Nacional Agraria La Molina, en Lima, Perú.

Los plantines de pimiento Páprika var. Papriqueen se obtuvieron del vivero SF Almácigos. Para la esterilización del suelo (mezcla de arena y suelo de chacra en proporción 1:2) se utilizó una autoclave a $120^{\circ} \mathrm{Cde}$ temperatura y 15 $\mathrm{lb}$ de presión por un periodo de una hora, evitando de esta manera la presencia de cualquier otro patógeno.

Las enmiendas evaluadas se obtuvieron de campos de cultivo de la UNALM, y de un aserradero comercial (Cuadro 1). Luego de secados fueron molidos, de manera que se uniformizó el tamaño de partícula a $2 \mathrm{~mm}$.

CUADRO 1. Enmiendas orgánicas utilizadas para el estudio del comportamiento del nematodo del nódulo Meloidogyne incognita (Kofoid\& White, 1919) Chitwood 1949, en páprika a nivel de invernadero.

\begin{tabular}{lcc}
\hline \multicolumn{1}{c}{ Nombre común / Especie } & Procedencia & Parte utilizada \\
\hline Aserrín de Cedro / Cedrelaodorata L. & Aserradero comercial - Sta. Anita & Tronco \\
Aserrín de Ulcumano /Podocarpusrospigliosii & Aserradero comercial - Sta. Anita & Tronco \\
Gallinaza / Gallusgallus & UNALM - Granja zootecnia & Guano \\
Guano de Vacuno / Bos Taurus & UNALM - Granja zootecnia & Guano \\
Broza de Espárrago / Asparagusofficinalis L. & UNALM - Unidad Experimental de Riego & Parte aérea \\
Rastrojo de Maíz / Zea mays L. & UNALM - Huerto & Follaje \\
Rastrojo de Leguminosa (Holantao) / Pisumsativum & UNALM - Huerto & Planta completa \\
L. var. Saccharatum & UNALM - Huerto & Parte aérea \\
Rastrojo de Nabo / Brassicacampestris L. spp. Rapa &
\end{tabular}

El sustrato usado para el trasplante de los plantines de Papriqueen resultó de la mezcla de 30 gramos de cada enmienda orgánica con $1 \mathrm{Kg}$ del suelo estéril. El trasplante se realizó a los 30 días de la siembra. Luego del trasplante se regaron los plantines de cada bolsa.

\section{Inoculación}

Para la inoculación fueron obtenidos huevos de Meloidogyne incognita de raíces de zapallo infestadas provenientes del valle de Huaral. Para su extracción se empleó el método propuesto por Hussey y Barker (1973). A tres días del trasplante se inoculó20000 huevos de Meloidogyne incognitaen cada bolsa conteniendo el sustrato, para los tratamientos Con Nematodos. Los plantines fueron regados (riegos ligeros y frecuentes) y cuidados todos los días.

A los 60 días del trasplante se evaluó el ensayo. Las variables evaluadas fueron: altura de planta, peso fresco de parte aérea, peso seco de parte aérea, peso fresco de raíz, volumen de raíz, grado de nodulación (de acuerdo a 3 escalas: Zeck, PIM y Baker), población de huevos, población de juveniles y población total de Meloidogyne incognita (Cuadros 2 y 3 ).

\section{Diseño Experimental}

En el ensayo se utilizó el programa computarizado SAS, versión 4.0 para realizar el análisis estadístico de los resultados obtenidos.

Se usó un Diseño Completo al Azar (DCA) con 9 tratamientos inoculados con nemátodos $(\mathrm{CN})$. Otros 9 tratamientos sin inocular ( $\mathrm{SN}$ ) fueron instalados con la finalidad de determinar si existe efecto fitotóxico de las enmiendas orgánicas. Se aplicó la prueba estadístico de Duncan. 


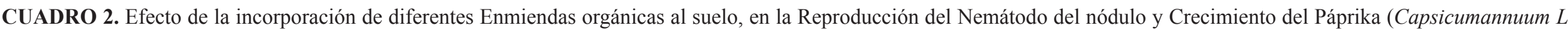
var.longum) inoculado con Meloidogyne incognita 2 meses después del trasplante.

\begin{tabular}{|c|c|c|c|c|c|c|c|c|c|c|c|c|}
\hline Clave & Tratamientos & $\begin{array}{l}\text { *Altura } \\
\text { de planta } \\
(\mathrm{cm})\end{array}$ & $\begin{array}{c}* \text { Peso } \\
\text { Fresco de } \\
\text { planta }(g)\end{array}$ & $\begin{array}{l}\text { * Peso Seco } \\
\text { de planta }(\mathrm{g})\end{array}$ & $\begin{array}{c}\text { Peso Fresco } \\
\text { de Raíz }\end{array}$ & $\begin{array}{l}\text { Volumen de } \\
\text { Raíz (cc) }\end{array}$ & $\begin{array}{c}\text { Grado de } \\
\text { Nodulacion } \\
(\text { Zeck })\end{array}$ & $\begin{array}{c}\text { Grado de } \\
\text { Nodulacion } \\
\text { (PIM) }\end{array}$ & $\begin{array}{c}\text { Grado de } \\
\text { Nodulacion } \\
\text { (Baker) }\end{array}$ & $\begin{array}{l}\text { Población } \\
\text { juvenil en } \\
\text { suelo }\end{array}$ & $\begin{array}{l}\text { Población de } \\
\text { huevos en } \\
\text { raíz }\end{array}$ & $\begin{array}{l}\text { Población } \\
\text { Total }\end{array}$ \\
\hline T1a & Testigo/Con & $44.0 \mathrm{AB}$ & $24.6 \mathrm{CB}$ & $2.3 \mathrm{AB}$ & $8.5 \mathrm{ABC}$ & $9.3 \mathrm{CB}$ & $5.5 \mathrm{AB}$ & $3.8 \mathrm{ABC}$ & $60.0 \mathrm{AB}$ & $200 \mathrm{~A}$ & $37600 \mathrm{AB}$ & $37800 \mathrm{~A}$ \\
\hline $\mathrm{T} 2 \mathrm{a}$ & $\begin{array}{l}\text { nematodos } \\
\text { Cedro / Con }\end{array}$ & $36.5 \mathrm{CD}$ & $8.6 \mathrm{E}$ & $1.4 \mathrm{D}$ & $6.6 \mathrm{C}$ & $7.8 \mathrm{CB}$ & $5.5 \mathrm{AB}$ & $4.3 \mathrm{~A}$ & $72.5 \mathrm{~A}$ & $500 \mathrm{~A}$ & $33200 \mathrm{AB}$ & $33700 \mathrm{~A}$ \\
\hline T3a & $\begin{array}{l}\text { nematodos } \\
\text { Ulcumano / Con }\end{array}$ & $40.0 \mathrm{CB}$ & $21.2 \mathrm{CD}$ & $2.1 \mathrm{CB}$ & $9.7 \mathrm{AB}$ & $10.1 \mathrm{AB}$ & $5.5 \mathrm{AB}$ & $3.8 \mathrm{ABC}$ & $55.0 \mathrm{AB}$ & $350 \mathrm{~A}$ & $37550 \mathrm{AB}$ & $37900 \mathrm{~A}$ \\
\hline $\mathrm{T} 4 \mathrm{a}$ & $\begin{array}{l}\text { nematodos } \\
\text { Gallinaza / Con }\end{array}$ & $46.8 \mathrm{~A}$ & $34.8 \mathrm{AB}$ & $2.6 \mathrm{~A}$ & $11.1 \mathrm{~A}$ & $12.3 \mathrm{~A}$ & $4.0 \mathrm{C}$ & $2.5 \mathrm{DC}$ & $27.5 \mathrm{CD}$ & $25 \mathrm{~A}$ & $30725 \mathrm{AB}$ & $30750 \mathrm{~A}$ \\
\hline T5a & $\begin{array}{l}\text { nematodos } \\
\text { Vacuno / Con }\end{array}$ & $42.8 \mathrm{AB}$ & $36.2 \mathrm{~A}$ & $2.7 \mathrm{~A}$ & $7.8 \mathrm{BC}$ & $6.8 \mathrm{C}$ & 4.8 ABC & $3.3 \mathrm{ABCD}$ & $60.0 \mathrm{AB}$ & $225 \mathrm{~A}$ & $19750 \mathrm{~B}$ & $19975 \mathrm{~A}$ \\
\hline T6a & $\begin{array}{l}\text { nematodos } \\
\text { Broza / Con }\end{array}$ & $41.3 \mathrm{CB}$ & 16.0 ED & $1.6 \mathrm{CD}$ & $7.4 \mathrm{BC}$ & $8.5 \mathrm{CB}$ & $5.8 \mathrm{~A}$ & 4.0 AB & $70.0 \mathrm{~A}$ & $75 \mathrm{~A}$ & $40900 \mathrm{~A}$ & $40975 \mathrm{~A}$ \\
\hline $\mathrm{T} 7 \mathrm{a}$ & $\begin{array}{l}\text { nematodos } \\
\text { Chala / Con }\end{array}$ & $37.3 \mathrm{CD}$ & $10.3 \mathrm{ED}$ & $1.4 \mathrm{D}$ & 7.1 BC & $8.8 \mathrm{CB}$ & $4.5 \mathrm{BC}$ & $2.8 \mathrm{BCD}$ & $42.5 \mathrm{BC}$ & $100 \mathrm{~A}$ & $26200 \mathrm{AB}$ & $26300 \mathrm{~A}$ \\
\hline T8a & $\begin{array}{l}\text { nematodos } \\
\text { Holantao / Con }\end{array}$ & $43.8 \mathrm{AB}$ & $32.8 \mathrm{AB}$ & $2.7 \mathrm{~A}$ & $10.6 \mathrm{~A}$ & $12.5 \mathrm{~A}$ & $2.5 \mathrm{D}$ & $2.0 \mathrm{DE}$ & $20.0 \mathrm{D}$ & 12:00 a.m. & $6525 \mathrm{C}$ & $6525 \mathrm{~B}$ \\
\hline Т9a & $\begin{array}{l}\text { nematodos } \\
\text { Nabo / Con } \\
\text { nematodos }\end{array}$ & $34.0 \mathrm{D}$ & $22.1 \mathrm{CD}$ & $1.9 \mathrm{CBD}$ & $6.2 \mathrm{C}$ & $7.3 \mathrm{CB}$ & $1.8 \mathrm{D}$ & $1.3 \mathrm{E}$ & $10.0 \mathrm{D}$ & 12:00 a.m. & $7150 \mathrm{C}$ & $7150 \mathrm{~B}$ \\
\hline
\end{tabular}

(*): Los valores consideran únicamente la parte aérea.

FUENTE: Elaboración propia 
CUADRO 3. Efecto de la incorporación de diferentes Enmiendas orgánicas en el desarrollo de Páprika, Capsicumannuum, 2 meses después del trasplante.

\begin{tabular}{|c|c|c|c|c|c|c|}
\hline Clave & Tratamientos & $\begin{array}{c}* \text { Altura } \\
\text { de planta }(\mathrm{cm}) \\
\end{array}$ & $\begin{array}{c}\text { * Peso Fresco de } \\
\text { planta }(\mathrm{g})\end{array}$ & $\begin{array}{c}\text { * Peso Seco de } \\
\text { planta }(\mathrm{g})\end{array}$ & $\begin{array}{r}\text { Peso Fre } \\
\text { de Raíz } \\
\end{array}$ & $\begin{array}{c}\text { Volumen de } \\
\text { Raíz (cc) } \\
\end{array}$ \\
\hline $\mathrm{T} 1 \mathrm{~b}$ & Testigo / Sin nematodos & $43.8 \mathrm{~B}$ & $31.1 \mathrm{AB}$ & $2.4 \mathrm{CD}$ & $7.1 \mathrm{BC}$ & $9.0 \mathrm{DC}$ \\
\hline $\mathrm{T} 2 \mathrm{~b}$ & Cedro / Sin nematodos & $41.0 \mathrm{~B}$ & 17.4 DE & $1.8 \mathrm{ED}$ & $9.5 \mathrm{~B}$ & $11.8 \mathrm{ABC}$ \\
\hline $\mathrm{T} 3 \mathrm{~b}$ & Ulcumano / Sin nematodos & $39.3 \mathrm{~B}$ & $24.0 \mathrm{CD}$ & $2.0 \mathrm{CD}$ & $8.4 \mathrm{BC}$ & $10.3 \mathrm{BCD}$ \\
\hline $\mathrm{T} 4 \mathrm{~b}$ & Gallinaza / Sin nematodos & $52.0 \mathrm{~A}$ & $33.5 \mathrm{~A}$ & $3.4 \mathrm{~A}$ & $12.8 \mathrm{~A}$ & $13.8 \mathrm{~A}$ \\
\hline $\mathrm{T} 5 \mathrm{~b}$ & Vacuno / Sin nematodos & $52.4 \mathrm{~A}$ & $37.7 \mathrm{~A}$ & $3.1 \mathrm{AB}$ & $8.3 \mathrm{BC}$ & $12.0 \mathrm{AB}$ \\
\hline T6b & Broza / Sin nematodos & $39.0 \mathrm{~B}$ & $21.0 \mathrm{CD}$ & $1.8 \mathrm{ED}$ & $7.0 \mathrm{C}$ & $9.8 \mathrm{BCD}$ \\
\hline $\mathrm{T} 7 \mathrm{~b}$ & Chala / Sin nematodos & $37.5 \mathrm{~B}$ & $12.9 \mathrm{E}$ & $1.4 \mathrm{E}$ & $7.0 \mathrm{C}$ & $8.0 \mathrm{D}$ \\
\hline $\mathrm{T} 8 \mathrm{~b}$ & Holantao/Sin nematodos & $41.0 \mathrm{~B}$ & $30.9 \mathrm{AB}$ & 2.6 CB & $11.7 \mathrm{~A}$ & $14.3 \mathrm{AB}$ \\
\hline T9b & Nabo / Sin nematodos & $42.0 \mathrm{~B}$ & $25.4 \mathrm{CB}$ & $3.1 \mathrm{AB}$ & $7.6 \mathrm{BC}$ & $9.0 \mathrm{DC}$ \\
\hline
\end{tabular}

(*): Los valores consideran únicamente la parte aérea. FUENTE: Elaboración propia

\section{Resultados y Discusión}

El análisis de variancia realizado a las diferentes variables evaluadas muestra que existen diferencias altamente significativas para los tratamientos: altura, peso fresco y peso seco de planta, volúmen y peso fresco de raíz y población total de Meloidogyne incognita.

Los tratamientos rastrojo de nabo (T9a) y rastrojo de holantao(T8a) mostraron ser efectivos en la reducción poblacional del nematodo del nódulo,en comparación con el tratamiento testigo inoculado (T1a) (Figura 1, Cuadro 2).

Para la enmienda rastrojo de nabo, esta reducción puede sustentarse en la actividad nematicida de sus compuestos azufrados, compuestos GLU e ITC (glucosinolatos e isotiocianatos, respectivamente) sobre numerosas especies como los Meloidogyne(Potter et al., 1998), o en el estrecho valor de su relación $\mathrm{C} / \mathrm{N}$ de 8 . Sin embargo, se encontró que la enmienda rastrojo de nabo afectó la altura de planta y generó amarillamiento del follaje del papriqueen, posiblemente por su rápida descomposición debido a su baja relación $\mathrm{C} / \mathrm{N}(\mathrm{C} / \mathrm{N}=8)$, la cual podría provocar problemas de deficiencia de nitrógeno(Rodríguez-Kabana et al., 1987).

El tratamiento rastrojo de holantao (T8a) también redujo la población final de Meloidogyne incognita, y tuvo un efecto positivo en el volúmen de raíz del páprika, que podría deberse a su adecuada relación C/N, coincidiendo con AkhtaryMalik (1999) en que de manera general las enmiendas orgánicas con estrecha relación carbono / nitrógeno, tales como las leguminosas, resultan en mejor control de nematodosque aquellas con amplia relación $\mathrm{C} / \mathrm{N}$.

Las demás enmiendas orgánicas: broza de espárrago con nemátodos (T6a),ulcumano con nematodos (T3a), cedro con nematodos (T2a), chala con nematodos (T7a), gallinaza con nematodos (T4a) y vacuno con nematodos
(T5a), resaltadas en color verde, no mostraron efectos favorables en la reducción de la población final de Meloidogyne incognita, resultando estadísticamente iguales al testigo(Figura 1, Cuadro 2).

Respecto a la nodulación, las tres escalas coinciden que el mejor control de nodulación de Meloidogyne incognita se obtiene incorporando las enmiendas orgánicas rastrojo de nabo y rastrojo de holantao(Figura 2). A su vez las escalas de nodulaciónZeck y Baker añaden al estiércol



Figura 1. Población final de Melougogyne incógnita a los 60 dias de la inoculación sobre diferentes sustratos provenientes de la mezcla con enmiendas orgánicas en Paprika var. Papriquen.

de gallinaza en el control de la nodulación (Cuadro 2). Además, el empleo del rastrojo de holantao y estiércol de gallinaza resultan de mayor beneficio para la planta pues las raíces del páprika mostraron mayor volúmen, tanto en las plantas inoculadas con Meloidogyne incognita, como en las que no fueron inoculadas.

Los resultados muestran que la densidad poblacional inoculada de Meloidogyne incognitaafectó los parámetros de crecimiento: altura de planta, peso seco de parte aérea y peso fresco de raíz de Papriqueen; mientras que elvolúmen de raíz no se vio afectado, e incluso tuvo 


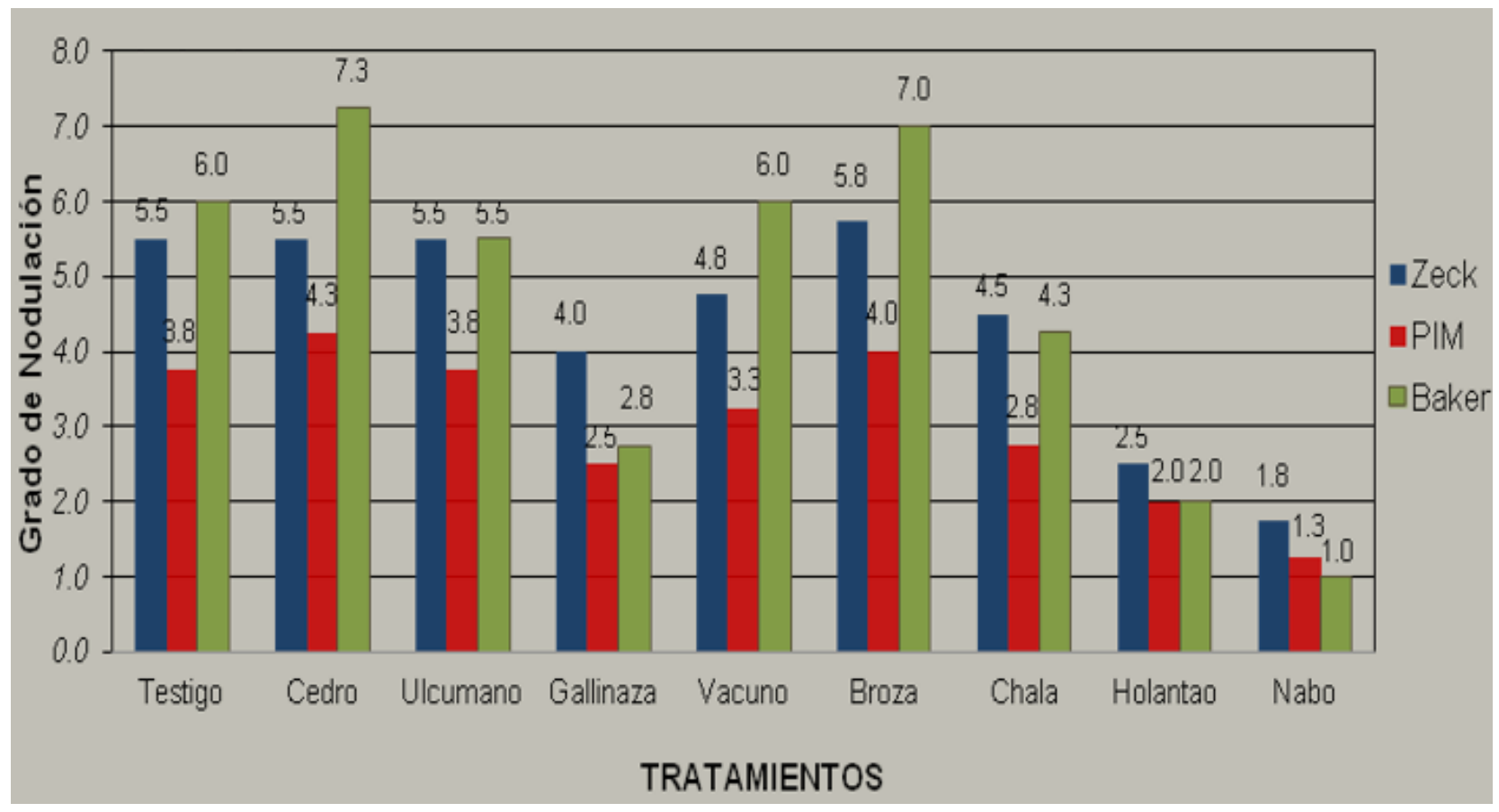

Figura 2. Comparativo de los grados de nodulación evaluados en el experimento, a los 60 días de la unoculación con MI en diferentes sustratos provenientes de la mezcla con enmiendas orgánicas en Paprika var. Papriqueen.

efectos positivos en parámetros de crecimiento como el peso fresco de planta Cuadro 2).

Respecto al parámetro altura de planta, se tuvo un efecto negativo de los tratamientos nabo con nematodos (T9a), cedro con nematodos (T2a) y chala con nematodos (T7a). Entre las posibles causas se tiene que para el caso del cedro con nematodos (T2a), el alto valor de su relación C/N (Cuadro 3) dificulta los procesos de descomposición de la M.O. por parte de los microrganismos del suelo, afectando la disponibilidad de nutrientes a las plantas y resultando en menor valor de altura de planta. Para los restos de cosecha (rastrojos de chala y nabo), la reducción de la altura de planta podría ser causada por un efecto de fitotoxicidad resultado de la alta dosis incorporada en el experimento.

Por el contrario, hubo una respuesta positiva con los tratamientos sin inoculación de Meloidogyne incognita, reportado con la incorporación de las enmiendas guano de vacuno (T5b) y guano de gallinaza (T4b), hipotéticamente debido a sus adecuados valores $\mathrm{C} / \mathrm{N}$ (menos de 20:1) que permitieron la rápida mineralización del nitrógeno en forma de $\mathrm{NH}+4$ o NO-3 para la absorción y la captación por las raíces de las plantas, consiguiendo mejora de los valores de altura de planta.

Respecto al parámetro peso seco de parte aérea de las plantas de Papriqueen, tanto en los tratamientos inoculados como en los que no fueron inoculados con Meloidogyne incognita, hubo un notorio efecto de reducción para las plantas que crecieron sobre el sustrato mezclado con la enmienda rastrojo de chala. Lo mismo se afirma para el parámetro peso fresco de parte aérea, donde las enmiendas orgánicas broza de espárrago, rastrojo de chala y aserrín de cedro presentaron un efecto de reducción del peso fresco tanto en los tratamientos inoculados como en los que no fueron inoculados con Meloidogyne incognita. De acá se deduce que la dosis incorporada para las enmiendas rastrojo de chala, broza de espárrago y aserrín de cedro pueden resultar fitotóxicas para las plantas de Papriqueen.

Como se menciona líneas arriba, la densidad poblacional inoculada de Meloidogyne incognitano afectó de manera negativa el volúmen de raíz del páprika, pero hubieron tratamientos que alcanzaron promedios de volúmen de raíz superiores al tratamiento testigo inoculado con Meloidogyne incognita (T1a); tales tratamientos fueron el guano de gallinaza (T4a) y rastrojo de holantao (T8a). Para la enmienda guano de gallinaza este incremento delvolúmen de raíz sería resultado de la producción de elementos nutritivos N, P2O5 y K2O asimilables por las raíces luego de la descomposición de esta enmienda orgánica en el suelo (Ruda de Schenquer E., Mongiello A., Acosta A., 2004). Además, debido a que la relación C / N en ambas enmiendas es menor de 20:1, la mineralización del nitrógeno para su captación y absorción por las raíces de las plantas se realizará de manera efectiva.

\section{Conclusiones}

Las enmiendas orgánicas rastrojo de holantao y rastrojo de nabo utilizadas en el presente estudio, han resultado eficaces para la reducción de las poblaciones y nodulación de Meloidogyne incognita. Sin embargo, el rastrojo de nabo tuvo la desventaja de alcanzar los más bajos valores promedio de altura de planta y peso fresco de raíz. 
Para este ensayo, las enmiendas orgánicas aserrín de cedro y rastrojo de chala, tuvieron efecto fitotóxico sobre las plantas de Papriqueen al ser incorporadas a dosis de $30 \mathrm{gr} / \mathrm{Kg}$ de sustrato, manifestándose en la coloración de hojas y el tamaño de planta.

\section{Literatura citada.}

Akhtar, M and Malik, A. 1999. Roles of organic soil amendments and soil organisms in the biological control of plant-parasitic nematodes: a review. BioresourceTechnology 74: 35-47 p.

Auger, J. y Thibout, E. 2004. Sustancias azufradas de los Allium y de las Crucíferas: potencial fitosanitario. In Biopesticidas de origen vegetal. Ediciones MundiPrensa. Madrid, ES. 75-92 p.

Badra, T. and Eligindi, DM. 1979. The relationship between phenolic content and Tylenchulus semipenetrans populations in nitrogen amended citrus plants. Revue de Nematologie 2, 161-164 p.

Craft, C. and Nelson, E. 1996. Microbial properties of composts that suppress damping-off and root rot of creeping bentgrass caused byPythiumgraminicola. Applied and Environmental Microbiology 62:1550-1557.

Hoitink, H. AJ and Boehm, MJ. 2001. Control biológico en comunidades microbianas del suelo: un fenómeno de dependencia de sustrato. Manejo Integrado de Plagas. Costa Rica. $N^{\circ}$ 62: 4-17.

Hoitink, H. AJ. and Grebus, M. 1994. Status of biological control of plant diseases with composts. Compost Science and utilization 2:6-12.

Hollis, JP. y Rodriguez-Kabana, R. 1966. Rapid kill of nemátodos in flooded soil. Phytopathology 56: 10151019.

Huber, DM. 1991. The use of fertilizers and organic amendments in the control of plant disease. In Ed. D Pimentel. Handbook of Pest Management in Agriculture. CRC Press. pp. 405-494.

Hussey, R. and Baker, KR. 1973. A Comparasion of Methods of Collecting inoculo of Meloidogyne spp. Including a New Technique. Plant. Dis. Rep. 57:10251028.

Muller, R. and Gooch, PS. 1982. Organic amendments in nematode control. An examination of the literature. Nematropica 12, 319-326.

Noble, R. y Coventry, E. 2005. Suppression of soilborne plant diseases with composts: A review. Biocontrol Science and Technology. 15:3-20.

Potter, MJ., Davis, K. y Rathjen, AJ. 1998. Suppressive impact of glucosinolates in Brassica vegetative tissues on root lesion nematode Pratyelenchusneglectus. J. Chem. Ecol., 24: 67-80
Rodriguez-Kabana, R., Morgan-Jones, G. y Chet, I. 1987. Biological control of nematodos: soil amendments and microbial antagonists. Plant and Soil 10, 237-247.

Ruda de Schenquer E; Mongiello, A; Acosta, A. 2004. Contaminación y salud del suelo. Ediciones UNL CETEA. Facultad de Ingeniería Química UNL. Santa Fé, República Argentina. 